\title{
MEASURE ALGEBRAS AND FUNCTIONS OF BOUNDED VARIATION ON IDEMPOTENT SEMIGROUPS ${ }^{1}$
}

\author{
BY STEPHEN E. NEWMAN
}

\section{Communicated by Gian-Carlo Rota, April 1, 1969}

Let $S$ be an abelian idempotent semigroup. Let $T$ be a semigroup of semicharacters on $S$ containing the identity semicharacter. A semicharacter on a semigroup $S$ is a nonzero, bounded, complex valued function on $S$ which is a semigroup homomorphism. A semicharacter on an idempotent semigroup is an idempotent function, and hence can assume only the values zero and one. We define $A_{f}=\{s \in S \mid f(s)=1\}$ and $J_{f}=\{s \in S \mid f(s)=0\}$ for each $f \in T$, and we denote by $A$ the Boolean algebra of subsets of $S$ generated by the sets $J_{f}(f \in T)$. If $X=\left\{f_{1}, \cdots, f_{n}\right\}$ is a finite subset of $T, \sigma \in T_{n}\left(T_{n}\right.$ denotes the Boolean algebra of all $n$-tuples of zeros and ones), we define

$$
B(X, \sigma)=\left\{\bigcap_{\sigma(i)=1} A_{f_{i}}\right\} \cap\left\{\bigcap_{\sigma(i)=0} J_{f_{i}}\right\} .
$$

Clearly, $A$ consists of finite unions of sets of the form (1). If $F$ is a function on $T, X$ and $\sigma$ are as above, we define an operator $L$ by

$$
L(X, \sigma) F=\sum_{\tau \in T_{n}} \mu(\sigma, \tau) F\left(\prod_{\tau \geq \sigma} f_{i}^{\tau(i)}\right)
$$

where

$$
\begin{aligned}
\mu(\sigma, \tau) & =(-1)^{|\tau|-|\sigma|} & & \tau \geqq \sigma, \\
& =0 & & \text { otherwise, }
\end{aligned}
$$

is the Möbius function for $T_{n}[3]$. Here $|\sigma|$ denotes the number of ones in the $n$-tuple $\sigma$. We call $F$ a function of bounded variation on $T$ if

$$
\sup _{\boldsymbol{X}} \sum_{\sigma \in T_{n}}|L(X, \sigma) F|<\infty,
$$

where the supremum is taken over finite subsets $X$ of $T$. The norm of $F$ is the number defined by (3). Finally, we say that $F$ is positive definite if

$$
L(X, \sigma) F \geqq 0
$$

1 These results were obtained in the author's doctoral dissertation written at the University of Utah under the direction of Professor Joseph L. Taylor. 
for all finite subsets $X$ of $T$ and all corresponding $\sigma$. Each of (2), (3), and (4) makes sense for functions on any abelian, idempotent semigroup with identity. It follows that the functions of bounded variation on any abelian, idempotent semigroup with identity form a partially ordered (in the obvious manner via (4)), normed linear space.

Let $\mu$ and $\nu$ be two finitely additive measures defined on the Boolean algebra $A$. A convolution product of $\mu$ and $\nu$ is defined by

$$
\mu * \nu(E)=\mu \times \nu\left(\alpha^{-1}(E)\right) \quad(E \in A),
$$

where $\alpha: S \times S \rightarrow S$ is the multiplication map on $S$. The measure $\mu \times \nu$ is defined on the Boolean algebra (not $\sigma$-algebra) of subsets of $S \times S$ generated by rectangles $E \times F(E, F \in A)$. The fact that $\alpha^{-1}(E)$ is a finite union of such rectangles for each $E \in A$ makes (5) meaningful.

We illustrate the above definitions with a simple but important example.

EXAMPLE 1. Let $S$ be the semigroup [0,1], under maximum multiplication $(x \cdot y=\max (x, y)$ for $x, y \in[0,1])$. Let $T=\left\{\chi_{[0, x]} \mid x \in[0,1]\right\}$ be the given semigroup of semicharacters on $S$. Note that $T$, under pointwise multiplication, is a semigroup isomorphic to $[0,1]$, under minimum. The Boolean algebra $A$ in this example consists of finite unions of left-open, right closed intervals and the single point 0 . A function on $T$ is of bounded variation in the sense of (3) if and only if it is of bounded variation (on $[0,1]$ ) in the classical sense. If $F$ is a function of bounded variation on $[0,1]$, then the norm of $F$ given by (3) is precisely the classical norm $(\|F\|=|F(0)|+V(F)$, where $V(F)$ is the total variation of $F$ ).

Detailed proofs of the following theorems will appear elsewhere.

Theorem 1 is established by purely algebraic methods involving the close relationship between the Boolean algebra $A$ and the semicharacters in $T$.

THEOREM 1. There exists an order-preserving isomorphism $\mu \rightarrow \hat{\mu}$ between the algebra of all finitely additive measures on $A$, under convolution multiplication, and the algebra of all functions on $T$, under pointwise multiplication. The function $\hat{\mu}$ is defined by $\hat{\mu}(f)=\mu\left(A_{f}\right)$ for each $f \in T$.

Theorem 2 follows trivially from Theorem 1.

THEOREM 2. The algebra $M(A)$ of all bounded, finitely additive measures on $A$, under convolution multiplication and total variation 
norm, is a Banach algebra. The algebra $B V(T)$ of all functions of bounded variation on $T$, under pointwise multiplication and bounded variation norm, is a Banach algebra. The map $\mu \rightarrow \mu$, defined in Theorem 1, maps $M(A)$ isomorphically and isometrically onto $B V(T)$.

The map $\mu \rightarrow \hat{\mu}$, in the setting of Example 1, is an extension of the relationship between bounded regular Borel measures and functions of bounded variation on $[0,1]$. The relationship between (1) and (2) is

$$
\mu(B(X, \sigma))=L(X, \sigma) \hat{\mu},
$$

where $\mu$ is a finitely additive measure on $A$. Thus the operator $L$ gives an inversion formula for $\mu \rightarrow \hat{\mu}$. For fixed $X$, this inversion formula is a simple application of combinatorial analysis involving the Möbius and zeta functions. The relevant combinatorial analysis can be found in Rota's paper on Möbius functions [3].

A Radon-Nikodým theorem can be proved in this context. Let $\nu$ and $\mu$ be measures on $A$ with $\nu$ absolutely continuous with respect to $\mu$. Let

$$
w_{X}=\sum_{\sigma \in T_{n}}(\nu(B(X, \sigma)) / \mu(B(X, \sigma))) \chi_{B(X, \sigma)}
$$

be a simple function on $S$ defined for each finite subset $X=\left\{f_{1}, \cdots f_{n}\right\}$ of $T$. We define $\nu(B(X, \sigma)) / \mu(B(X, \sigma))=0$ whenever $\mu(B(X, \sigma))=0$ (and hence $\nu(B(X, \sigma))=0)$. A corresponding measure $\nu_{X}$ is given by $\nu_{X}(E)=\int_{E} w_{X} d \mu(E \in A)$. The measures $\nu_{X}$ form a net, ordered by $X \leqq Y$ if $X \subset Y$. The proof of the following theorem relies heavily on Darst's Radon-Nikodým theorem [1].

THEOREM 3. Let $\nu$ and $\mu$ be two bounded measures on $A$ with $\mu$ positive and $\nu$ absolutely continuous with respect to $\mu$. Then the net $\left\{\nu_{X}\right\}$ defined above converges to $\nu$ in total variation norm, and the net $\left\{w_{X}\right\}$ of simple functions converges in $\mu$-measure.

Theorem 3 allows us to compute the Radon-Nikodým net $\left\{w_{X}\right\}$ directly from the functions $\hat{\nu}$ and $\hat{\mu}$. In fact, it follows from (6) and (7) that

$$
w_{X}=\sum_{\sigma}(L(X, \sigma) \hat{\nu} / L(X, \sigma) \hat{\mu}) \chi_{B(X, \sigma)} .
$$

The quotient $L(X, \sigma) \hat{\nu} / L(X, \sigma) \hat{\mu}$ plays the same role as the difference quotient $(G(x)-G(y)) /(F(x)-F(y))$ does in defining the derivative $d G / d F$ for functions on $[0,1]$. We point out that the net $\left\{w_{x}\right\}$ is the 
closest thing to a Radon-Nikodym derivative possible in our setting since the measures $\nu$ and $\mu$ are only finitely additive. If $\nu$ and $\mu$ are extendable to countably additive measures on some $\sigma$-algebra over $S$ containing $A$, then we can prove using [1] that $\left\{w_{X}\right\}$ converges in $L^{1}(\mu)$ norm to the actual Radon-Nikodým derivative $d \nu / d \mu$.

Our next theorem states that the algebra $B V(T)$ of all functions of bounded variation on an abelian idempotent semigroup $T$ with identity is a semisimple, commutative convolution measure algebra in the sense of [4]. A convolution measure algebra is roughly a lattice ordered Banach space with a multiplication which makes it a Banach algebra and relates appropriately to the norm and the order. A precise definition can be found in [4]. Our proof is straightforward. We first show the existence of an order preserving, linear isometry of $B V(T)$ onto the ordered Banach space of all bounded, finitely additive measures on a Boolean algebra. We use this isometry and Darst's Radon-Nikodym theorem [1] to verify a technical condition given in the definition of a convolution measure algebra. The norm inequality

$$
\|F G\| \leqq\|F\|\|G\| \quad(F, G \in B V(T))
$$

is proved directly using the Möbius and zeta functions. These remarks combine to yield

THEOREM 4. Let $T$ be an abelian idempotent semigroup with identity. Then the algebra $B V(T)$ of all functions of bounded variation on $T$, under pointwise multiplication of functions, is a semisimple, commutative convolution measure algebra.

Theorem 4 enables us to apply Taylor's structure theory for semisimple, commutative convolution measure algebras [4] to the algebra $B V(T)$. Accordingly, there exists a compact topological semigroup $S$, called the structure semigroup of $B V(T)$, and an embedding $F \rightarrow F_{S}$ of $B V(T)$ into $M(S)$ (the bounded regular Borel measures on $S$ under convolution) such that each complex homomorphism of $B V(T)$ has the form $h_{f}(F)=\int_{S} f d F_{S}$ for some $f \in \hat{S}$ (the continuous semicharacters on $S)$. Let $M$ be the image of the map $F \rightarrow F_{S}$. The point evaluation map $h_{x}\left(h_{x}(F)=F(x)\right.$ for $\left.x \in T, F \in B V(T)\right)$ is a complex homomorphism of $B V(T)$. Let $f_{x}$ be the semicharacter in $B V(T)$ which corresponds to the homomorphism $h_{x}$; in this manner, each $x \in T$ is identified with some $f_{x} \in \hat{S}$. We can now prove the following theorem.

THEOREM 5. Let $T$ be an abelian idempotent semigroup with identity. Then there exists a compact topological semigroup $S$, an L-subalgebra $M$ of $M(S)$, and a semigroup isomorphism $x \rightarrow f_{x}$ of $T$ into $\hat{S}$ such that: 
1. Each complex homomorphism $h$ of $M$ has the form $h(\mu)=\int_{s} f d \mu$ $=\hat{\mu}(f)$ for some $f \in \hat{S}$.

2. If we consider $T \subset \hat{S}$ via the embedding $x \rightarrow f_{x}$, then the map $\left.\mu \rightarrow \hat{p}\right|_{T}$ is an isomorphism and order preserving isometry of $M$ onto $B V(T)$.

The semigroup $T$ can be embedded in the semigroup (under pointwise multiplication) $\hat{S}$ as an idempotent subsemigroup which clearly separates points in $B V(T)$. However, $\hat{S}$ itself need not be idempotent. A modification of an example given in [2] provides a counterexample.

\section{REFERENCES}

1. R. B. Darst, $A$ decomposition of finitely additive set functions, J. Reine Angew. Math. 210 (1962), 31-37.

2. S. E. Newman, Measure algebras on idempotent semigroups, Pacific J. Math. (to appear).

3. G.-C. Rota, On the foundations of combinatorial theory: I. Theory of Möbius functions, Z. Wahrscheinlichkeitstheorie und Verw. Gebiete 2 (1964), 340-368.

4. J. L. Taylor, The structure of convolution measure algebras, Trans. Amer. Math. Soc. 119 (1965), 150-166.

UNIVERSity OF Missouri, St. LoUIS, Missouri 63121 